\title{
Ueber die Richtungen der grossen Axen der Cometenbahnen.
}

\author{
(A uszug aus einer der k. Akademie der Wissenschaften zu Wien am I8. November i886 \\ vorgelegten Abhandlung nebst einigen kleinen Zusätzen.)
}

Im Folgenden wird gezeigt, dass die mitunter als Beweis für die Eigenbewegung unseres Sonnensystems und den extrasolaren Ursprung der Cometen herbeigezogene Tendenz der grossen Axen der Comètenbahnen, sich in der Nähe der heliocentrischen Längen $270^{\circ}$ und $90^{\circ}$ dichter als an anderen Stellen anzusammeln, durch Verhältnisse terrestrischer Natur erklärt werden kann, nämlich durch die Umstände, welche der Auffindung von Cometen am günstigsten sind.

Von vorneherein besteht kein Grund gegen die Annahme, dass die Perihelpunkte sämmtlicher Cometenbahnen, von der Sonne aus gesehen, an der Himmelssphäre nahezu gleichmässig vertheilt sind. Dagegen ist die Wahrscheinlichkeit, von der Erde aus bemerkt zu werden, nicht für alle Cometen dieselbe. Im Allgemeinen wird ein Comet um so leichter zu unserer Wahrnehmung gelangen, je mehr er den folgenden drei Forderungen entspricht: I) soll er selbst ein ansehnlicher Körper sein; 2) soll er der Sonne nahe kommen, damit seine absolute Lichtstärke eine grosse wird; 3) soll die Möglichkeit geboten sein, den Cometen unter einem grossen Gesichtswinkel wahrzunehmen und von seinem Licht möglichst viel aufzufangen, er soll also auch der Erde nahe kommen. Die erste Forderung ist an die physische Beschaffenheit der Cometen gebunden und muss hier ausser Acht gelassen werden; die anderen hängen aber von den Bahnelementen $\mathrm{ab}$, und zwar die zweite von der Periheldistanz, die dritte von der Gesammtheit der Elemente in Verbindung mit dem Ort der Erde.

Am günstigsten muss sich die Sichtbarkeit eines Cometen, also auch die Wahrscheinlichkeit seiner Auffindung, offenbar dann gestalten, wenn gleichzeitig, soweit es möglich ist, sowohl die eine als die andere Bedingung erfullt wird, d. h. wenn ein Comet zur Zeit seiner grössten Lichtstärke, also in der Nähe des Perihels, auch in die Nähe der Erde gelangt. In diesen Falle erscheint er uns nämlich in der grössten überhaupt möglichen Helligkeit. Der Perihelpunkt eines solchen Cometen liegt, von der Sonne aus gesehen, in der der Erde zugewandten Parthie des Himmels. Ist die zur Zeit des Periheldurchganges des Cometen stattfindende heliocentrische Länge der Erde $L_{0} \pm 180^{\circ}$, ferner die heliocentrische Länge und Breite des Perihelpunktes $l$ und $b$, so lässt sich diese Bedingung so ausdrücken, dass $b$ klein und $l=L_{0} \pm 180^{\circ}$ ist; sie gilt sowohl, wenn $q<\mathrm{I}$, somit die Elongation des Cometen von der Sonne während der grössten Helligkeit $<90^{\circ}$, als auch, wenn $q>1$, somit die Elongation des Cometen zur Zeit des Perihels $>90^{\circ}$ ist. Diesen Schlüssen zufolge sollen also unter den bekannten Cometen jene überwiegen, welche dieser doppelten Bedingung genügen, d. h. somit jene Cometen, die während ihrer Sonnennähe auch in die Erdnähe kommen.

Es giebt aber auch Cometen - und hierher gehören namentlich die mit sehr kleiner Periheldistanz - welche nicht zur Zeit des Perihels in die Erdnähe gelangen können, sondern nur auf dem Hinweg zum Perihel oder auf dem
Rückweg vom Perihel sich der Erde nähern und während dieser Annäherung für uns am besten sichtbar sind. Für diese kehrt sich der zweite Theil der obigen Bedingung in das Gegentheil um, d. h. wir nehmen unter den Cometen mit kleiner Periheldistanz vorzugsweise solche wahr, die der Bedingung $l=L_{0}$ genügen.

Nach den bisherigen Erwägungen besteht also bei den relativ meisten Cometen ein Zusammenhang zwischen der Perihelzeit und der Perihellänge in der Weise, dass die Perihellängen der zu unserer Wahrnehmung gelangenden Cometen im Laufe des Jahres mit der Erde um die Sonne wandern. Diese Eigenschaft kann auch so ausgesprochen werden, dass die heliocentrischen Projectionen der Perihelpunkte nicht weit von den zugehörigen heliocentrischen Positionen der Erde liegen, dass somit sowohl der Winkel $b$, als auch $l-\left(L_{v} \pm 180^{\circ}\right)$ und für Cometen mit kleiner Periheldistanz der Winkel $l-L_{0}$ nicht gross, beispielsweise nur $30^{\circ}$ ist.

Wann tritt nun dieser Zusammenhang zwischen Perihelzeit und Perihellänge am stärksten zu 'Tage? Offenbar dann, wenn unter den in einer bestimmten Jahreszeit durch das Perihel gehenden Cometen die meisten zu unserer Wahrnehmung gelangen können, wenn es also möglich ist, die Cometen in relativ kleinen Elongationen von der Sonne zu beobachten, wo ja in der Regel ihre grösste Helligkeitsentfaltung stattfindet. Diese Gelegenheit bietet uns im Sommer die Circumpolargegend des Himmels, weil wir hier Cometen in kleinen Elongationen von der Sonne auch in der Nacht, ja sogar um Mitternacht beobachten können, und aus diesem Grunde können von den im Sommer durch das Perihel gehenden Cometen die meisten gefunden werden. Da die heliocentrische Länge der Erde während des Sommers in der Nähe von $270^{\circ}$ ist, so werden also Perihellängen in der Nähe von $270^{\circ}$ überwiegen.

In jeder anderen Jahreszeit ist uns der Umkreis der Sonne um so weniger erreichbar, je südlicher die Sonne steht, am wenigsten also im Winter. Dennoch rufen aber auch einige im Winter durch das Perihel gehende Cometen ein Uebergewicht hervor, und zwar diejenigen, deren Periheldistanz gross, nämlich gegen I und darüber hinaus ist. Solche Cometen kommen unserer Erde meist in sehr grossen Elongationen, ja sogar in der Opposition nahe und werden am ehesten gefunden, wenn die zur Sonne in Opposition befindliche Himmelsgegend ihren höchsten Stand hat, also im Winter. Es liefert also auch der Winter ein Maximum der Perihellängen und zwar bei $90^{\circ}$, welches aber schwächer als das bei $270^{\circ}$ ist, weil es hauptsächlich von den in geringerer Zahl vorhandenen Cometen mit grösserer Periheldistanz herrührt.

Die Helligkeit der Sommernächte kann nicht als Einwand gegen diese Behauptungen gelten; denn wenn Cometen, wie es ja wiederholt geschieht, in der Morgen- und Abenddämmerung entdeckt werden, so können sie in der durch die Mittsommersonne verursachten Helligkeit ebenso gut oder 
gar noch leichter gefunden werden, weil hier Gelegenheit geboten ist, einen grossen Theil des Sonnenumkreises durch längere Zeit, also mit Musse zu durchforschen. Auch die vielfachen Trübungen der Atmosphäre im Winter fallen nicht schwer ins Gewicht, weil die bedeutende Länge der Nächte Gelegenheit giebt, vorübergehende Aufheiterungen öfter als in kurzen Nächten auszunutzen.

Die Cometen mit kleiner Periheldistanz bieten uns für $l=L_{v}$ die günstigsten Sichtbarkeitsverhältnisse, und diese sind somit denen der bisher behandelten Cometen entgegengesetzt; sie verstärken also im Winter die Lingen bei $270^{\circ}$ und im Sommer die Längen bei $90^{\circ}$. (Siehe auch R. Lehmann-Filhés , Ueber die Cometen und Meteorströme mit klciner Periheldistanz \& A. N. Bd. 96.) Dieses Uebergewicht kann aber nicht auffallig hervortreten, weil die Zahl solcher Cometen gering ist.

Wir haben bis jetzt die Nordhemisphäre der Erde im Auge gehabt. Wenn wir nun fragen, wie sich die Südhemisphäre zu diesem Thema verhält, so sehen wir gleich, dass durch den geänderten Standpunkt der Beobachter an dem Wesen der Thatsache, dass längs der Meridiane $90^{\circ}$ und $270^{\circ}$ eine Anhäufung der Cometenperihele stattfindet, eigentlich nichts geändert wird, sondern dass nur eine Verschiebung um $180^{\circ}$ geschieht. Der Sommer der Südhalbkugel wird nämlich die Perihele bei $l=90^{\circ}$, der Winter bei $l=270^{\circ}$ häufen; entsprechende Verhältnisse müssten sich, falls die Cometenentdeckungen auf der Südhalbkugel zahlreicher werden sollten, auch bei den Cometen mit kleiner Periheldistanz zeigen. Beide Hemisphären wirken also in demselben Sinne, beide verstärken die Maxima bei $90^{\circ}$ und $270^{\circ}$. Nur die Entdeckungen in den Tropengegenden, für welche keine Hemisphäre des Himmels ein Uebergewicht hat, wïrden wahrscheinlich jede Stelle der Ekliptik nahe gleichmässig mit Cometen-Perihelien besetzen. Ebenso können auch Cometen, die sich während ihrer Sichtbarkeit nicht weit vom Aequator entfernen, z. B. I 886 VII und $\mathrm{I887}_{7}$ IV kein Uebergewicht von bestimmten Perihellängen hervorrufen.

Ich habe nun, um die Richtigkeit meiner Erwägungen an den bekannten Cometen zu prüfen, nach den Formeln

$$
\begin{aligned}
\cos b \cos (l-\Omega) & =\cos (\pi-\Omega) \\
\cos b \sin (l-\Omega) & =\sin (\pi-\Omega) \cos i \\
\sin b & =\sin (\pi-\Omega) \sin i
\end{aligned}
$$

die Perihelpositionen $l$ und $b$ berechnet und dabei auch die ersten Erscheinungen der periodischen Cometen aufgenommen; auf diese Weise sind gerade 300 Cometen zusammengekommen, deren letzter $1886 \mathrm{~V}$ ist. Daraus geht nun vor allem die Thatsache hervor, dass die heliocentrische Breite der Perihele $b$ der bekannten Cometen wirklich meist klein, in der Nähe von $30^{\circ}$, und nur ausnahmsweise gross, über $60^{\circ}$ ist; es finden sich nämlich:

Nördliche Perihele.

$$
\begin{array}{rr}
0^{\circ} \text { und }+30^{\circ} & 88 \\
++30,+60 & 65 \\
+60,+90 & 25 \\
\hline 78
\end{array}
$$

Südliche Perihele.

$$
\begin{array}{rrrr}
\text { zw. } 0^{\circ} \text { und } & -30^{\circ} & 85 \\
-30 & -60 & 26 \\
-60 & -90 & 11
\end{array}
$$

Was den Zusammenhang zwischen $T$ und $l$ betrifft, so gehört theoretisch $z u$ jedem Grad der heliocentrischen Perihellänge $l$ ein bestimmter Periheltag $T$, nämlich jener, der die Relation $l=L_{0} \pm 180^{\circ}$ erfüllt, somit zu jedem Bogen von $30^{\circ}$ Länge ein bestimmter Perihelmonat (natürlich ohne Rücksicht auf ein bestimmtes Jahr). Ich lasse jedoch, um kleine Zufälligkeiten mehr zu verwischen, ausser dem Perihelmonat auch noch seine beiden Nachbarmonate mitstimmen, bringe also jeden Bogen von $30^{\circ}$ Länge mit einem ,Perihel-Vierteljahre in Verbindung, nämlich:

$$
\begin{aligned}
& l=10^{\circ}-40^{\circ} \text { mit September, October, November, } \\
& l=40^{\circ}-70^{\circ} \text { mit October, November, December, } \\
& \text { u. s. w. }
\end{aligned}
$$

\begin{tabular}{|c|c|c|c|c|c|c|c|c|c|c|c|c|c|c|c|c|}
\hline 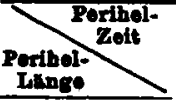 & 㞼 & 点 & 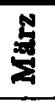 & 胥 & 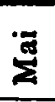 & 点 & 㕦 & $\stackrel{\circ 0}{2}$ & 莒 & む் & 产 & 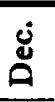 & $S$ & $1 / 4 S$ & $M$ & $D$ \\
\hline $10^{\circ}=40^{\circ}$ & 2 & I & I & I & I & 3 & 一 & 4 & 6 & 2 & 4 & - & 25 & $61 / 4$ & 12 & $+5^{3 / 4}$ \\
\hline $40 \cdot 70$ & 4 & 2 & 3 & 2 & - & I & I & - & $\mathbf{I}$ & 3 & 10 & 2 & 29 & $7^{1 / 4}$ & I 5 & $+7^{3 / 4}$ \\
\hline $70-100$ & 10 & I & I & - & 2 & $\mathbf{I}$ & 2 & - & 6 & 2 & 4 & 3 & 32 & 8 & 17 & +9 \\
\hline $100 \cdot 130$ & 4 & 2 & 2 & 3 & I & 2 & 2 & - & I & 6 & $\mathbf{I}$ & 4 & 28 & 7 & 10 & +3 \\
\hline $130 \cdot 160$ & $\mathbf{I}$ & 6 & $\mathbf{I}$ & 3 & -- & - & - & 一 & - & 2 & - & 5 & 18 & $41 / 2$ & 8 & $+31 / 2$ \\
\hline $160 \cdot 190$ & 2 & 2 & I & I & 2 & 2 & 3 & $I$ & - & 2 & $\mathbf{I}$ & 3 & 20 & 5 & 4 & $-I$ \\
\hline $190-220$ & $\mathbf{I}$ & 3 & 2 & 3 & 4 & I & - & - & $\mathbf{I}$ & - & $\mathbf{I}$ & - & 16 & 4 & 9 & +5 \\
\hline $220-250$ & - & - & 2 & 4 & 4 & 6 & 3 & 2 & 4 & $I$ & 3 & - & 29 & $71 / 4$ & 14 & $+63 / 4$ \\
\hline $250 \cdot 280$ & - & I & 2 & 4 & 3 & 8 & 4 & $\mathbf{x}$ & 5 & 3 & 5 & 2 & $3^{8}$ & $91 / 2$ & 15 & $+51 / 2$ \\
\hline $280-310$ & 2 & $\mathbf{x}$ & 2 & I & 3 & 4 & 4 & 2 & $\mathbf{I}$ & I & 3 & 2 & 26 & $61_{i 2}$ & 10 & $+3^{1 / 2}$ \\
\hline $310 \cdot 340$ & $\mathbf{I}$ & 一 & $x$ & - & - & I & 3 & 7 & 2 & $\mathbf{I}$ & - & 4 & 20 & & 12 & \\
\hline $340 \cdot 10$ & - & 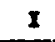 & - & 2 & $\mathbf{I}$ & $\mathbf{I}$ & I & 3 & 5 & 2 & $I$ & 2 & 19 & $4 \%$ & 10 & $+51 \%$ \\
\hline Summe & 27 & 20 & 18 & 24 & $2 \pi$ & 30 & 23 & 20 & 32 & 25 & $\overline{33}$ & 27 & 300 & 75 & 136 & 6I \\
\hline
\end{tabular}

Um einen klaren Ueberblick zu erlangen, habe ich auf Grund meines Verzeichnisses die Anzahl der in jedem Monat erfolgten Periheldurchgänge und die zugehörigen Perihellängen, in Gruppen zu je 30 Graden abgetheilt, in ein Schema zusammengestellt. Die jedem Perihelmonat zukornmende Zahl, die theoretisch unter allen in derselben Horizontalreihe stehenden Zahlen entweder für sich selbst oder doch in Verbindung mit ihren Nachbarzahlen das relative Uebergewicht haben soll, ist durch den Druck hervorgehoben. 
Die Columne $\$$ giebt die Anzahl aller in das jeweilige $3 \circ$ gradige Längenintervall gehörenden Periheldurchgänge, $1 / 4 S$ den vierten 'Theil derselben, zeigt also an, wie viele im Durchschnitt auf ein Vierteljahr entfallen. In der Columne $M$ steht die Anzahl der dem Perihelvierteljahr entstammenden Periheldurchgänge, d. h. die Summe aus der hervorgehobenen und ihren beiden benachbarten Zahlen.

Diese Zahlen $M$ sind nun, wie es die Forderung $l=L_{0} \pm 180^{\circ}$ erheischt, mit einziger Ausnahme der zu $l=160^{\circ}-190^{\circ}$ gehörenden Zahl (deren Minus übrigens wegen des Plus der oben und unten stehenden beiden Nachbarzahlen wenig zu bedeuten hat) durchgehends grösser als die Zablen der Columne $1 / 4 . S$, denen sie nur dann im Durchschnitt gleich sein könnten, wenn die Perihellängen nicht an die Perihelzeiten gebunden, sondern zufällig, also nahe gleichmässig vertheilt wären. Die Zahlen der letzten Columne $D$ geben die Differenzen $M-1 /{ }_{4} S$ und haben um so grösseres Gewicht, je grösser sie selbst und je grösser die zugehörigen Gesammtsummen $S$, oder eigentlich $1 / 4, S$ sind. Die Endsummen lehren, dass, während von 300 Periheldurchgängen auf ein Vierteljahr im Durchschnitt 75 entfallen, dem Perihelvierteljahr 136 entstammen, also ein Ueberschuss von $6 \mathrm{r}$ Cometen.

Betrachtet man das Schema genauer, so sieht man, dass zur Bildung des Maximums in der Nähe von $270^{\circ}$ nur die Sommermonate beitragen, welche sonst im ganzen Jahre spärlich vertreten sind, und dass ein Ausfall oder auch nur eine Verringerung dieser Zahlen das Uebergewicht dieser Perihellängen aufheben würde, ebenso zeigt sich, dass das Maximum bei $90^{\circ}$ durch die Wintermonate hervorgerufen wird.

Um mich zu versichern, dass die in unserem Sommer durch das Perihel gehenden Cometen wirklich der Mehrzahl nach in unserer Circumpolargegend des Himmels gefunden werden, habe ich für die Cometen, deren Perihellänge zwischen $240^{\circ}$ und $300^{\circ}$ liegt, nachgesehen, in welchem Sternbild sie entdeckt oder überhaupt beobachtet wurden. $\mathrm{Da}$ sind nun die Sternbilder Cassiopeia, Camelopardalis, Draco, Cepheus, Perseus, Auriga, Lynx und Ursa major am häufigsten vertreten, also durchgehends solche, die im Sornmer eine geringe Elongation von der Sonne (im Durchschnitt etwa $30^{\circ}$ ) haben und für unsere Breiten auch in der vollen Nacht über dem Horizont sind.

Uebrigens ist es gar nicht nothwendig, dass die Cometen in geringen Elongationen von der Sonne gefunden werden; wichtig ist nur, dass sie in geringen Elongationen ihre grösste Helligkeit erreichen. So wären die Cometen 1874 III, I886 I und 1886 II, auch wenn sie nicht schon mehrere Monate vor dem Perihel, wo sie noch lichtschwach waren, entdeckt worden wären, sicher in der Nähe des Peribels, also in geringer Elongation von der Sonne gesehen worden. Massgebend für das Princip ist eben jene Himmelsgegend und jene Zeit, in welcher die Auffindung mit der geringsten Anstrengung geschieht.

Es muss bemerkt werden, dass bei Ermittelung der Zahlen $M$ und $D$ nur auf die Relation $l=L_{0} \pm 180^{\circ}$ Rücksicht genommen und, die für Cometen mit kleiner Periheldistanz geltende Relation $\ell=L_{0}$ wegen der geringen Zahl dieser Gestirne ausser Acht gelassen worden ist.
Uebrigens sind nicht gerade immer Cometen mit kleiner Periheldistanz diejenigen, welche blos auf einem Parabelast und zwar an einer vom Perihel weit entfernten Stelle beobachtet werden, sondern dieser Umstand kann sich ausnahmsweise auch bei anderen Cometen und zwar besonders bei solchen mit sehr grosser Periheldistanz zeigen, weil bei diesen wegen der bedeutenden Bahndimensionen eine Beobachtung auf beiden Parabelästen gewöhnlich nicht möglich ist.

Auch muss hervorgehoben werden, dass bei Anlegung des Schemas nur der Standpunkt der Nordhemisphäre in Betracht gezogen worden ist. In Wirklichkeit sind aber manche Cometen ausschliesslich der Südhemisphäre zuzılzählen, sprechen also nicht für die Relation $l=L_{0} \pm 180^{\circ}$, sondern für $l=L_{0}$. Durch diese Unterscheidung würde sich die Bestätigung meiner Folgerungen noch etwas besser gestalten, doch lässt sich eine consequente Auswahl solcher Cometen nicht strenge durchführen, namentlich nicht bei denjenigen, die stets in kleinen Declinationen geblieben sind. Im Allgemeinen wäre wohl ein Comet zu jener Hemisphäre zu zählen, auf welcher er seine grösste Helligkeit gezeigt hat.

Es ist also nicht nur durch blosse Ueberlegungen, sondern auch durch die Thatsachen dargethan, dass die dichtere Ansammlung der grossen Axen der Cometenbahnen längs der heliocentrischen Meridiane $90^{\circ}$ und $270^{\circ}$ auf terrestrische Verhältnisse zurückgeführt werden kann. Man hat diese Ansammlung bisweilen als ein der Radiation der Sternschnuppen analoges Phänomen hingestellt und den Umstand nicht beachtet, dass in diesem Falle nur in der Nähe des Apex der Sonnenbewegung ein Maximum, in der entgegengesetzten Gegend aber ein Minimum sein müsste, während doch in der Wirklichkeit jede dieser beiden Gegenden ein Maximum von Perihelien resp. Aphelien aufweist.

Der in der Regel vorhandene Zusammenhang zwischen $T$ und $l$ hat zur Folge, dass wir unter normalen Verhältnissen beinahe gezwungen sind, in einer bestimmten Jahreszeit hauptsächlich Cometen mit einer bestimmten Bahnaxenrichtung wahrzunehmen und demnach wäre sogar die Mög. lichkeit vorhanden, nach der Entdeckung eines neuen Cometen, falls dieselbe wirklich nicht weit von der Epoche der günstigsten Sichtbarkeitsverhältnisse, also auch nicht allzu weit vom Perihel entfernt ist, sofort die Richtung der Bahnaxe mit einer Genauigkeit von etwa $\pm 30^{\circ}$ anzugeben. Wie weit die Regel die Ausnahmen überwiegt, kann im Perihel-Schema aus den Zahlen $D$ ersehen werden.

In früheren Jahrhunderten, wo man Cometen nicht suchte, sondern sich durch das Auftauchen derselben über. raschen liess, hätte ein solches Unternehmen nur in den wenigsten Fällen ein wesentlich unrichtiges Resultat geliefert, jetzt aber, wo man mit immer stärkeren Hülfsmitteln nach ihnen ausschaut, können Cometen schon mehrere Monate vor ihrer grössten Helligkeit zu unserer Kenntniss gebracht werden, andererseits Objecte, die selbst während der grössten Helligkeit noch immer lichtschwach bleiben und ohne besondere Nachforschung unbemerkt weiter ziehen würden, für unser Cometenverzeichniss gewonnen werden.

Fragt man, ob durch diese verschärften Nachsuchungen das hier aufgestellte Princip vielleicht nach und nach unhaltbar wird, so muss die Antwort im Allgemeinen wohl 
verneinend ausfallen. Bringt nämlich das Teleskop Cometen lange vor ihrer grössten Helligkeit zu unserer Kenntniss, so sind solche Cometen später noch leichter zu finden, weil die Wahrscheinlichkeit ihrer Entdeckung um so mehr wächst, je näher das Perihel oder überhaupt die Epoche der grössten Helligkeit rïckt; und massgebend ist ja nicht die Zeit der Entdeckung, sondern die der grössten Helligkeit. Lehrt uns das Teleskop Cometen kennen, die wegen ihrer Lichtschwäche ohne Anwendung kräftiger optischer Hülfsmittel nicht $\mathrm{zu}$ unserer Kenntniss gelangen würden, so ist zu beachten, dass auch mit dem Fernrohr unter den lichtschwachen und lichtschwach bleibenden Cometen gerade wieder solche am leichtesten zu finden sind, welche den hier aufgestellten Bedingungen entsprechen, während andere Cometen von gleicher Lichtschwäche um so mehr unbemerkt bleiben müssen, je weniger sie diese Bedingungen erfüllen. Eine Abschwächung des Princips könnte allerdings durch solche Cometen bewirkt werden, welche mit Hülfe des Fernrohrs nur bei geringer Helligkeit gesehen werden, bei grösserer Helligkeit aber wegen kleiner Elongation von der Sonne nicht mehr wahrzunehmen sind; solche Fälle sind aber selten.

Zum Schluss seien die hier gefundenen zwei Beziehungen nochmals angeführt.

Erstens wurde dargethan, dass unter allen $\mathrm{zu}$ unserer Wahrnehmung gelangenden Cometen jene überwiegen, für welche sowohl $b$ als $l-L_{v} \pm 180^{\circ}$, für Cometen mit kleiner Periheldistanz $l-L_{0}$ eine mässige Grösse ist. Diese Beziehung gilt für die Erde uberhaupt, sie wird somit durch Rücksichtnahme auf eine bestimmte Hemisphäre nicht alterirt.

Zweitens wurde gezeigt, dass unter den dieser Bedingung entsprechenden Cometen, so lange die Beobachter in etwas höheren, sei es nördlichen oder südlichen geographischen Breiten sind, wieder diejenigen überwiegen, deren heliocentrische Perihellänge $l$ in der Nähe von $90^{\circ}$ oder $270^{\circ}$ liegt. Diese Eigenheit ist also durch den Standpunkt der Beobachter verursacht.

Es sollen nun auch für die neuen Cometen, welche in das obige Perihel-Schema noch nicht aufgenommen

Wiener Sternwarte 1888 Sept. 29. worden sind, die hierher gehörenden Zahlen mitgetheilt werden. Für drei Cometen ist nicht $l-L_{0} \pm 180^{\circ}$, sondern $l-L_{0}$ gegeben und zwar für $1887 \mathrm{I}$ wegen seiner kleinen Periheldistanz, für 1886 VIII und 1888 III aber darum, weil sie, obwohl mit grösseren Periheldistanzen versehen, dennoch erst auf einer vom Perihel schon weit entfernten Strecke und zwar beide auf dem Rückweg vom Perihel beobachtet werden konnten, eine Eigenthümlichkeit, die sie also mit Cometen von kleiner Periheldistanz gemein haben.

\begin{tabular}{|c|c|c|c|c|c|c|}
\hline Comet & $T$ & $q$ & $l$ & $b$ & $\begin{array}{l}l-L_{0} \\
\pm 180^{\circ}\end{array}$ & $l-L_{0}$ \\
\hline & Nov. 22 & 1.00 & $8^{\circ}$ & $-2^{\circ}$ & $-53^{\circ}$ & 一 \\
\hline & Nov. 28 & 1.48 & 261 & +32 & - & $+14^{\circ}$ \\
\hline I 886 IX & Dec. 16 & 0.66 & 65 & +78 & -20 & - \\
\hline 18 & Jan. I I & 0.01 & 282 & $+3^{8}$ & - & -9 \\
\hline 37 II & März I 7 & 1.63 & 105 & +20 & -71 & - \\
\hline III & März 28 & 1.01 & 106 & +23 & -82 & - \\
\hline I 887 IV & Juni 16 & 1.39 & 260 & +5 & -6 & - \\
\hline 188 & Oct. 8 & 1.20 & 142 & +40 & +126 & - \\
\hline & März r 7 & 0.70 & 245 & & +68 & 一 \\
\hline I 888 III & Juli 3 I & 0.90 & 126 & +56 & - & \\
\hline
\end{tabular}

Wie man sieht, hat die heliocentrische Winkeldifferenz zwischen dem Perihel und der zugehörigen Position der Erde in der Regel eine mässige Grösse. Ist die Längendifferenz $l-L_{0} \pm 180^{\circ}$ grösser, so ist gewöhnlich der Breitenunterschied $b$ kleiner und umgekehrt. Am aufallend. sten gegen die Regel spricht die grosse Längendifferenz von $126^{\circ}$, welche der Comet $1887 \mathrm{~V}$, der Olbers'sche Comet, aufweist. Sie zeigt eben, dass die Sichtbarkeitsverhältnisse des Cometen in dieser Erscheinung recht ungünstig waren; am besten sind sie, wenn er im Februar durch das Perihel geht. Die übrigen Cometen, bei denen die Längen- oder Breiten-Differenz mässige Beträge übersteigt, sind meistens an sich ansehnliche Körper gewesen, z. B. I886 IX, 1888 I und in geringem Grade auch $1887 \mathrm{II}$; diese sind also nicht so sehr wegen ihrer günstigen Stellung als vielmehr wegen ihrer physischen Beschaffenheit auffallende Objecte gewesen.

\section{F. Holetschek.}

Neuer Comet Barnard Oct. 30. Ein Telegramm aus Boston meldet:

A comet was discovered by Barnard Oct. 31.0399 Gr. M. T. RA. app. $=145^{\circ} 50^{\circ} 33^{\prime \prime}$ PD. app. $=105^{\circ} 18^{\circ} 52^{\prime \prime}$. Daily motion $+23^{\prime}$ in RA., $-9^{\prime}$ in PD. Physical appearance: Slightly elongated, diameter $I^{\prime}$, 1 I mag. or fainter, strong central condensation.

Neuer Planet (28I).*) Prof. Weiss in Wien meldet telegraphisch:

Planet Palisa 31. October $13^{\mathrm{h}} 29^{\mathrm{m}} \mathrm{x}$ M. Z. Wien AR. app. $=30^{\circ} 4 \mathrm{I}^{\circ} 33^{\prime \prime}$ PD. app. $=76^{\circ} 25^{\circ} 31^{\prime \prime}$. Tägl. Bew. $-17^{\circ}$ in AR., $+\mathbf{r}^{\prime}$ in PD. Grösse $1 \mathbf{2}^{\mathbf{m}}$.

*) Ueber einen anderen, von Dr. Palisa am 29. October entdeckten Planeten, der zunächst mit (255) Oppavia für identisch gehalten wurde, aber vermuthlich neu ist, folgt Mittheilung in nächster Nummer. K'r.

\section{Inhalt:}

Zu Nr. 2364-65. W. Luther. Beobachtuugen von Cometen und kleinen Planeten. r13. - F. Terby. La rainure près de Godin. I35. - F. Holitschek. Ueber die Richtungen der grossen Axen der Cometenbahnen. 137. - Neuer Comet Barnard Oct. 30. 143. - Neuer Planet (281). 143.

Geschlussen 1888 Nor. 3. Heraugeber: A. Kr u eger. Druck ron C. Sc ha id t, C. F. Mohr Naohf. Expedition: Sternwarte in Kiel. 